\title{
Hypertensive emergency with discrepancy between arterial line and sphygmomanometer
}

\author{
Wyatt McGilvery $^{1 *}$, Spencer Salazar ${ }^{1}$, Michelle Uttaburanont ${ }^{2}$ and Maciej Witkos ${ }^{1}$ \\ ${ }^{1}$ Department of Emergency Medicine, Loma Linda University Medical Center, USA \\ ${ }^{2}$ Rancho Family Medical Group, USA
}

\begin{abstract}
The authors present a case in which a 28-year-old male was brought to the emergency department via ambulance with a chief complaint of uncontrolled hypertension pertaining to home measurements of systolic pressures in the low 200's mmHg and subsequent shortness of breath. EMS treated the patient ASA and Nitroglycerine, in the Emergency Department the patient received, esmolol, and fentanyl. Despite aggressive treatments to lower blood pressure, the patient's mentation was decreasing rapidly. Blood pressures taken by both manual and automatic sphygmomanometers were indicative of hypertensive pressures between $200-220 \mathrm{mmHg}$ systolic and 110-125 mm diastolic. However, it became apparent that these pressures were not accurate when an arterial line was placed reading systolic pressures well over $300 \mathrm{mmHg}$. The discrepancy between these modalities of obtaining blood pressure were disconcerting, as the arterial line pressure matched the patient's symptomology, and the sequela of hypertension.
\end{abstract}

\section{Introduction}

Hypertensive emergency is defined as an elevated blood pressure (BP) with accompanying end-organ damage. In general, hypertensive emergencies tend to occur in patients at systolic pressures $>180 \mathrm{mmHg}$ and diastolic pressures $>120 \mathrm{mmHg}$. However, there is no definite threshold, as some patients may exhibit end-organ damage even when below these numbers, if there is an acute rise in blood pressure. Other patients might not exhibit extreme organ damage even when pressures reach exceptionally high limits. The Narloch Brandstater 1995 study of blood pressure during heavy weightlifting recorded a maximum systolic blood pressure of 370 in male athletes during strenuous resistance exercises via Arterial-Line measurements [1-3]. While the patient should always be treated based on the symptomology and physical findings combined - it is important to ensure accurate information is collected, specifically the accuracy of instantaneous blood pressure readings. As in this case, it is possible that sphygmomanometers need to be replaced with arterial lines in patients with hypertensive emergencies. This case study has been submitted to, and approved by Loma Linda University Health's Institutional Review Board (IRB) in order to ensure all ethical criteria have been met.

\section{Case report}

The authors present a case in which a 28 -year-old male was brought to the emergency department via ambulance with a chief complaint of uncontrolled hypertension and subsequent shortness of breath. This patient has a past medical history of drug abuse, end stage renal disease on dialysis, and hypertension. The patient has had reoccurring hospital admissions for hypertensive emergency with acute encephalopathy and hyperkalemia which were usually successfully treated with nifedipine, nitroglycerine drips and emergent hemodialysis. On this presentation, the patient reported initial home blood pressure measurements of 160 $\mathrm{mmHg}$ systolic and rapidly increasing to the low 200's mmHg. At this time, the patient began experiencing acute shortness of breath and chest pain.
EMS Pre-hospital care 3 doses of nitroglycerine (4mg via sublingual), $325 \mathrm{mg}$ acetylsalicylic acid (ASA), and $4 \mathrm{mg}$ of intravenous morphine for the complaint of chest pain. Prehospital EKG showed ST elevation in V1, and the second EKG repeated in the emergency department was absent of any ST elevations. The patient claimed to be compliant with Monday, Wednesday, Friday hemodialysis, as well as all blood pressure medications.

Upon arrival to emergency department the patient was placed on full telemetry. On initial physical assessment, the patient appeared to have waxing and waning mental status and was noted with a systolic blood pressure reaching up to $160 \mathrm{mmHg}$. Ensuing early vitals showed a normal heart rate at 80 beats per minute, oral temperature of 98.2 degrees F, pulse oximetry of $97-100 \%$ on 15 liters per minute oxygen via nonrebreather. Automatic sphygmomanometery showed severe hypertension in the $200-220 \mathrm{mmHg}$ systolic, $110-125 \mathrm{mmHg}$ diastolic. Despite initial aggressive treatment, the patient's blood pressure began to rise into the 230's mmHg systolic, with a subsequent decrease in mental status. At this time, it was decided to place an arterial line into the patient's right radial artery for continuous arterial pressure measurements.

It was disconcerting to note that the arterial line measurements indicated the patients' blood pressure was elevated far beyond what the sphygmomanometer was claiming - 320/155 mmHg with a good waveform. The strikingly large discrepancy between the arterial line and the automated blood pressure cuff continued. The arterial line continued to reach levels between $280-320 \mathrm{mmHg}$ systolic, while

*Correspondence to: Wyatt McGilvery, Department of Emergency Medicine, Loma Linda University Medical Center, USA, E-mail: wyattmcgilvery@gmail.com

Key words: hypertensive emergency, arterial line, hypertension, calibration

Received: March 26, 2020; Accepted: April 20, 2020; Published: April 22, 2020 


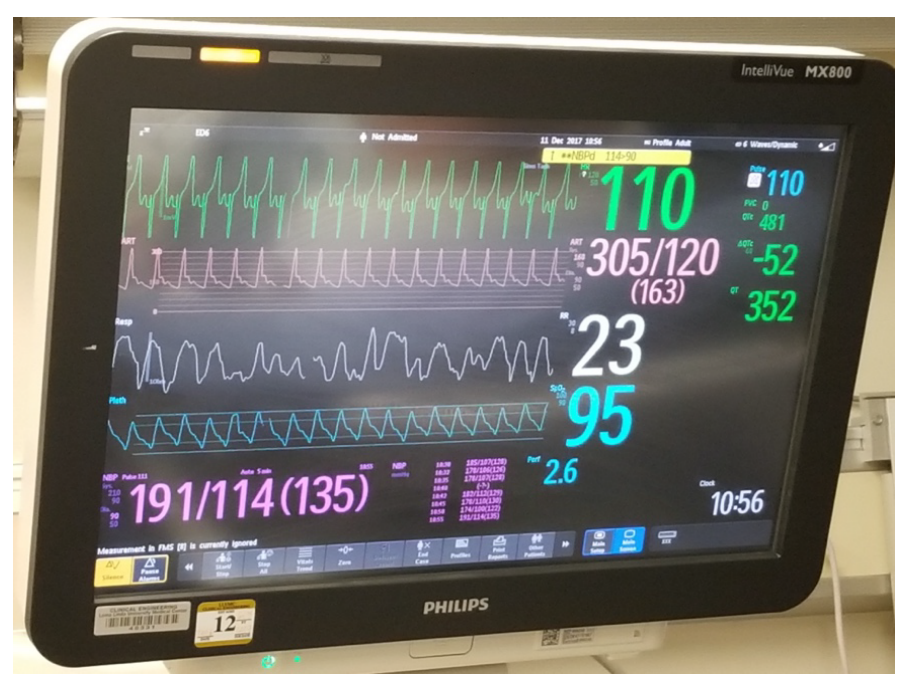

Figure 1. Full Telemetry monitoring displaying the patient's instantaneous vitals at 1056 . Patient was considered to be in sinus tachycardia and tachypneic with a respiratory rate of 23 breaths per minute. However, the monitor also displays the extreme discrepancy between arterial, and automated sphygmomanometer blood pressure readings, including the large discrepancy in the patients mean arterial pressure

the automated blood pressure cuff would only reach up to 220-230's $\mathrm{mmHg}$ systolic (Figure 1). All other vitals remained stable, although abnormal. Evidently, there was an average discrepancy of $36.03 \%$ between the arterial line and the automatic blood pressure cuff machine. It is important to note that multiple different sphygmomanometers were used, including emergency department staff utilizing a manual sphygmomanometer. All Sphygmomanometers continued to display a large discrepancy in blood pressure compared to the arterial line when the patient remained in an extreme hypertensive state.

Another dose of nitroglycerine $(0.6 \mathrm{mg})$ was administered, followed by fentanyl ( $25 \mathrm{mcg}$ muscular injection) for the continuing chest pain, and an esmolol infusion $2500 \mathrm{mg} / 250 \mathrm{~mL}$ (titrated at $200 \mathrm{mcg} / \mathrm{kg} / \mathrm{min}$ ) with addition of nitroglycerine (titrated at $0.5 \mathrm{mcg} / \mathrm{kg} / \mathrm{min}$ ) was given. Once the blood pressure began to decrease, we observed the accuracy between the arterial line and the automated cuff improve, as well as the patient's chest pain. Once the hypertensive state of the patient was stabilized, he was admitted to the medical intensive care unit for further monitoring and treatment.

The patient was subsequently weaned off intravenous (IV) medications and switched to oral medications. The patient still required IV hydralazine and labetalol intermittently throughout his admission. Once normotensive vitals

\section{Discussion}

Hypertensive emergency is defined as an elevated blood pressure (BP) with accompanying end-organ damage. In general, hypertensive emergencies tend to occur in patients at systolic pressures $>180 \mathrm{mmHg}$ and diastolic pressures $>120 \mathrm{mmHg}$. However, there is no definite threshold as some patients may exhibit end-organ damage even when below these numbers, particularly if there is an acute rise in blood pressure. Additionally, patients with chronic hypertension may have severely elevated pressures, and yet show no signs of end-organ damage. The Narloch Brandstater 1995 study of blood pressure during heavy weightlifting recorded a maximum systolic blood pressure of $370 \mathrm{mmHg}$ in male athletes during strenuous resistance exercises via Arterial-Line measurements [3]. This is the highest recorded Blood pressure to date found in literature. The average maximum BP in the
Narloch Brandstater study was found to be $320 \mathrm{mmHg}$, which was our patient's maximum blood pressure at rest. Care must be taken to focus on the patient's symptoms and clinical presentations and not solely blood pressure numbers. This principle is demonstrated in our case. We did not advance to an arterial line because the patient's blood pressure was elevated on the automated blood pressure cuff. The arterial line was placed due to the continued clinical decompensation of the patient. Only when the arterial line was placed, was it noted that the there was a large discrepancy between the blood pressure readings from the arterial line, and the automated sphygmomanometer. The patient's clinical presentation and symptomology correlated with the arterial line readings and directed our treatment plan. It is known that blood pressure measurement with an automated cuff can be inaccurate in particular instances. It has been observed that in patients with atrial fibrillation and other heart rhythm irregularities, blood pressure taken with an automated cuff is not reliable [4-6]. This needs to be considered in cases where the data collected is not correlating with a patient's clinical picture. It may be that extreme hypertension also causes inaccurate blood pressure readings in an automated cuff.

The hypertensive emergency is often classified based on the organ system being affected. The "target organs" most often affected include the brain, heart, kidneys, and large arteries. Specifically, cerebral infarction (39 percent) and acute pulmonary edema (25 percent) appear to be the most common hypertensive emergencies [7]. Because different "target organs" are affected, the presentation of the hypertensive emergency patient can vary.

Therapy will often be geared towards the specific hypertensive emergency and organs being affected. Generally, it is recommended that mean arterial pressure (MAP) be reduced gradually by approximately 10 to 25 percent in the first hour and by a further 5 to 15 percent over the next 23 hours [2]. However, lowering the patient's Blood pressure should be done with caution as lowering numbers too rapidly can result in ischemic damage in certain patient populations.

A common neurological hypertensive emergency, that is demonstrated in this particular case, is hypertensive encephalopathy. The symptoms in hypertensive encephalopathy will often subside once the blood pressure is addressed, as was the case with our patient. The general guidelines for lowering blood pressure is to proceed at 10 to 25 percent in the first hour followed by a more gradual decrase [5]. The most common medications used in this scenario are nicardipine, clevidipine, fenoldopam and nitroprusside. Intravenous beta-blockers are also an acceptable means of lowering the pressure, especially if the patient has concerning cardiac symptoms. Nitroprusside should be used with caution as patients can start exhibiting signs of toxicity at doses of $2 \mathrm{mcg} / \mathrm{kg} / \mathrm{min}$ or greater.

\section{Conclusion}

Treatment should be tailored to the specific hypertensive emergency, and the focus of treatment should be based on clinical picture, not only blood pressure numbers alone. In the case of our patient, he continued to decline clinically despite high, but stable, automatic blood pressure readings. Subsequent insertion and monitoring of blood pressure in our patient with an arterial line proved to show a significant discrepancy in blood pressure readings. The arterial line provided more accurate clinical data to help guide treatment with increased precision. This case sparks the question as to when should the use of arterial lines be utilized? As stated before, it is important to utilize the physical exams, findings, and symptomology to treat the hypertensive emergency patient. However, if the symptomology does not initially match the 
patient's blood pressure readings, perhaps it would be best to place an arterial line when it comes to the patient's blood pressure, as well as blood pressure trends when medications or interventions are utilized. More randomized controlled trial evidence is needed to better establish guidelines regarding hypertensive emergencies and to better determine which antihypertensive therapies best improve patient outcomes [4,5].

\section{References}

1. Alshami A, Romero C, Avila A, Varon J (2018) Management of hypertensive crises in the elderly. Journal of Geriatric Cardiology 15(7): 504-512. [Crossref]

2. Elliott WJ (2006) Clinical features in the management of selected hypertensive emergencies. Progress in Cardiovascular Diseases 48(5): 316-325. [Crossref]
3. Narloch JA, Brandstater ME (1995) Influence of breathing technique on arterial blood pressure during heavy weight lifting. Archives of Physical Medicine and Rehabilitation 76(5):457-62.

4. Perez MI, Musini VM (2008) Pharmacological interventions for hypertensive emergencies: a Cochrane systematic review. Journal of Human Hypertension 22(9): 596. [Crossref]

5. Salvetti M, Paini A, Bertacchini F, Stassaldi D, Aggiusti C, et al. (2018) Acute blood pressure elevation: Therapeutic approach. Pharmacological Research 130: 180-190. [Crossref]

6. Shayne PH, Pitts SR (2003) Severely increased blood pressure in the emergency department. Annals of Emergency Medicine 41(4): 513-529.

7. Zampaglione B, Pascale C, Marchisio M, Cavallo-Perin P (1996) Hypertensive urgencies and emergencies: prevalence and clinical presentation. Hypertension 27(1): 144-147.

Copyright: $\left(\mathrm{C}^{2020}\right.$ McGilvery W. This is an open-access article distributed under the terms of the Creative Commons Attribution License, which permits unrestricted use, distribution, and reproduction in any medium, provided the original author and source are credited. 